\title{
PA-035 FEASIBILITY OF IMPLEMENTING A CONTINUOUS HOUSEHOLD MALARIA INDICATOR SURVEY IN RARIEDA SUB-COUNTY, SIAYA COUNTY, WESTERN KENYA
}

Brian Seda, ${ }^{1}$ Titus Kwambai, ${ }^{1}$ Phelix Jangu, ${ }^{1}$ Aaron Samuels, ${ }^{2}$ Feike ter Kuile, ${ }^{3}$ Simon Kariuki . ' ${ }^{1}$ KEMRI-CGHR, Kenya; ${ }^{2}$ CDC, United States of America; ${ }^{3}$ LSTM, United Kingdom

\subsection{6/bmjgh-2016-000260.71}

Background Malaria transmission in Siaya County is high and perennial with peak transmission in May-July and October-November. The Kenya Medical Research Institute (KEMRI) and Centers for Disease Control and Prevention (CDC) have historically conducted malaria surveillance in western Kenya during peak malaria transmission season through annual population-based cross-sectional surveys. However, it may be important to characterise the seasonal spatial variation in malaria transmission for surveillance purposes, to monitor malaria interventions, and to inform decisions for changing and targeting malaria control strategies. We describe the feasibility of implementing a continuous malaria indicator survey (cMIS) for malaria surveillance in Rarieda sub-County, Kenya.

Methods All households in the study area were GPS-mapped and household members enumerated. Community interviewers were trained and assessed in collecting blood for malaria rapid diagnostic tests (RDT), preparation of dried blood spots on Whatman 903 filter paper, preparing blood smears, and performing HemoCue ${ }^{\circledR}$ tests for haemoglobin determination. Community interviewers were also trained to provide appropriate treatment with antimalarials and haematinics based on test results. They visited a random subset of 5 houses in the study area each week and collected and transmitted data in real-time throughout the year.

Results Four trained community interviewers visited a total of 1041 compounds, and consented and tested a total of 4714 participants for malaria by RDT in year one. Approximately $27 \%$ were positive for malaria by RDT and were offered treatment.

Conclusions A large number of compounds were visited by few staff in year one. This suggests that cMIS may be a viable way to perform year-round surveillance, and to offer malaria testing and treatment in the community with minimal staff. Further analysis of results from cMIS and comparisons to existing surveillance platforms are warranted to determine if cMIS can provide accurate estimates of malaria case burdens throughout the year. 